\title{
THE EXTENT OF DEFINABLE SCALES
}

\author{
BY DONALD A. MARTIN, YIANNIS N. MOSCHOVAKIS AND JOHN R. STEEL ${ }^{1}$
}

The notion of a scale on a pointset is implicit in the classical proof of the Kondo uniformization theorem for $\Sigma_{2}^{1}$ sets and in the uniformization theorem of Martin and Solovay [2] for $\Sigma_{3}^{1}$ sets, from the assumption that measurable cardinals exist. It was formally isolated in Moschovakis [3], where it was shown (granting projective determinacy) that all projective sets admit projective scales and hence, projective sets can be projectively uniformized. Soon after that it became clear that sets which admit definable scales have many desirable properties in addition to uniformization, and the natural problem arose to determine exactly which sets admit definable scales.

Assuming hyperprojective determinacy, Moschovakis [5] proved that every inductive set admits an inductive scale and asked the obvious next question, whether coinductive sets admit definable scales. This appeared to be a crucial test of the strength of determinacy hypotheses and we included it in the list of Victoria Delfino problems in [1].

We will show here (in outline) that under strong determinacy hypotheses, every coinductive set indeed admits a definable scale - which is typically substantially more complicated than the given set. We will also solve completely the general problem of the extent of scales within the model $L(R)$ of sets constructible above the continuum: if $L(R)$ satisfies the axiom of full determinacy (AD), then within $L(R)$, a set admits a scale if and only if it is a $\Sigma_{1}^{2}$ set (this settles a conjecture of Solovay). One of the lemmas in the latter proof is a simple and elegant characterization of the $\Sigma_{1}^{2}$ sets in $L(R)$ which is proved in classical ZermeloFraenkel set theory and appears to be an analog of the Shoenfield absoluteness theorem (for $\Sigma_{2}^{\mathbf{1}}$ ) for this model.

It is convenient to assume the notation and terminology of [6], but we will repeat some of the basic definitions that we need in order to make this note more easily comprehensible. Full proofs and related results will be published elsewhere.

1. Scales on coinductive sets. As usual, a space is any product

$$
X=X_{1} \times \cdots \times X_{n}
$$

Received by the editors October 8, 1981.

1980 Mathematics Subject Classification. Primary 03E15, 03E60; Secondary 04A15, $03 \mathrm{D} 60$.

${ }^{1}$ Work supported in part by NSF Grant MCS 78-02989. 
of copies of $\omega$ (the integers) and $N={ }^{\omega} \omega$ (the "reals") and a pointset is any subset $P \subseteq X$ of a space. A semiscale on $P$ is any sequence $\bar{\varphi}=\left\langle\varphi_{i}: i \in \omega\right\rangle$ of norms $\varphi_{i}: P \rightarrow$ ordinals on $P$, such that if $x_{0}, x_{1}, \ldots \in P, \lim _{n \rightarrow \infty} x_{n}=x$ and if for each $i$ the sequence of ordinals $\left\langle\varphi_{i}\left(x_{n}\right): n \in \omega\right\rangle$ is ultimately constant, then $x \in P ; \bar{\varphi}$ is a scale if the same assumptions imply that for all $i, \varphi_{i}(x) \leqslant \lim _{n \rightarrow \infty} \varphi_{i}\left(x_{n}\right)$.

For any pointclass (collection of pointsets) $\Gamma$, a norm $\varphi: P \rightarrow$ ordinals is a $\Gamma$-norm if both relations $\leqslant_{\varphi}^{*},<_{\varphi}^{*}$ are in $\Gamma$, where

$$
\begin{aligned}
& x \leqslant_{\varphi}^{*} y \Longleftrightarrow x \in P \&[y \notin P \vee \varphi(x) \leqslant \varphi(y)], \\
& x<_{\varphi}^{*} y \Longleftrightarrow x \in P \&[y \notin P \vee \varphi(x)<\varphi(y)] .
\end{aligned}
$$

If $\left\langle\Gamma_{i}: i \in \omega\right\rangle$ is a sequence of pointclasses with specified universal sets $G_{i} \subseteq N \times$ $X \times X(i \in \omega)$ and $\bar{\varphi}=\left\langle\varphi_{i}: i \in \omega\right\rangle$ is a sequence of norms (e.g. a semiscale on some $P \subseteq X)$, we will naturally say that $\varphi_{i}$ is $a \Gamma_{i}$-norm uniformly if we can effectively find for each $i$ codes for $\leqslant_{\varphi_{i}}^{*}$ and $<_{\varphi_{i}}^{*}$ in $\Gamma_{i}$.

A pointset $P \subseteq X$ is inductive if there is some $R \subseteq \omega^{2}$ such that (1) $P(x) \Longleftrightarrow\left\{\left(\forall \alpha_{0}\right)\left(\exists \alpha_{1}\right)\left(\forall \alpha_{2}\right)\left(\exists \alpha_{3}\right) \ldots\right\}(\exists t) R\left(\left\langle\alpha_{0}(t), \ldots, \bar{\alpha}_{t-1}(t)\right), \bar{x}(t)\right)$, where the $\alpha_{i}$ vary over $N,\langle\rangle, \bar{\alpha}$ and $\bar{x}$ and denote the usual (recursive) codings of tuples and the infinite string of alternating quantifiers is interpreted in the usual way via an open game on $N$. If we define the real-game quantifier $\mathrm{S}^{2}$ by its action

$$
\left(\supset^{2} \alpha\right) P(x, \alpha) \Longleftrightarrow\left\{\left(\forall \alpha_{0}\right)\left(\exists \alpha_{1}\right)\left(\forall \alpha_{2}\right) \ldots\right\} P\left(x,\left\langle\alpha_{0}, \alpha_{1}, \ldots\right\rangle\right),
$$

then the inductive pointsets are clearly those in the class $S^{2} \Sigma_{1}^{0}\left(=\right.$ all $\supset^{2} P$, with $\left.P \in \Sigma_{1}^{0}\right)$. These are exactly the pointsets which are $\Sigma_{1}$-definable over $N^{+}=R^{+}=$ the smallest admissible set which contains $N$ or $R=$ the true reals or again those pointsets which can be defined by positive elementary inductions over $N$, as in [4] and [6, Chapter 7].

Finally, let $\Sigma_{0}^{*}$ be the pointclass of all Boolean combinations of inductive and coinductive sets and for $n>0$, call $P$ a $\Sigma_{n}^{*}$-pointset if $P$ satisfies

$$
P(x) \Longleftrightarrow\left(\exists \alpha_{1}\right)\left(\forall \alpha_{2}\right)\left(\exists \alpha_{3}\right) \ldots\left(Q_{n} \alpha_{n}\right) R\left(x, \alpha_{1}, \alpha_{2}, \ldots, \alpha_{n}\right),
$$

where $R$ is in $\Sigma_{0}^{*}$. Let $\Sigma^{*}=\bigcup_{n} \Sigma_{n}^{*}$.

Theorem A (Moschovakis). If every $\Sigma^{*}$ game is determined, then every coinductive set $P$ admits a scale $\bar{\varphi}=\left\langle\varphi_{i}: i \in \omega\right\rangle$ such that each $\varphi_{i}$ is a $\Sigma_{i}^{*}$-norm, uniformly.

Outline of Proof. By the dual of (1) above, if $P$ is coinductive, then we have an equivalence of the form

(1*) $P(x) \Longleftrightarrow\left\{\left(\exists \alpha_{0}\right)\left(\forall \alpha_{1}\right)\left(\exists \alpha_{2}\right) \ldots\right\}(\forall t) R\left(\left\langle\bar{\alpha}_{0}(t), \bar{\alpha}_{1}(t), \ldots, \bar{\alpha}_{t-1}(t)\right\rangle, \bar{x}(t)\right)$. 
For each even $n$, put

$$
\begin{gathered}
P_{n}\left(x, \alpha_{0}, \ldots, \alpha_{n-1}\right) \Longleftrightarrow\left\{\left(\exists \alpha_{n}\right)\left(\forall \alpha_{n+1}\right)\left(\exists \alpha_{n+2}\right) \ldots\right\} \\
(\forall t) R\left(\left\langle\alpha_{0}(t), \bar{\alpha}_{1}(t), \ldots, \bar{\alpha}_{t-1}(t)\right\rangle, \bar{x}(t)\right),
\end{gathered}
$$

and for each odd $n$, put

$$
\begin{gathered}
P_{n}\left(x, \alpha_{0}, \ldots, \alpha_{n-1}\right) \Longleftrightarrow\left\{\left(\forall \alpha_{n}\right)\left(\exists \alpha_{n+1}\right)\left(\forall \alpha_{n+2}\right) \ldots\right\} \\
(\forall t) R\left(\left\langle\alpha_{0}(t), \bar{\alpha}_{1}(t), \ldots, \bar{\alpha}_{t-1}(t)\right\rangle, \bar{x}(t)\right),
\end{gathered}
$$

so that $P=P_{0}$ and

(2) $P_{n}\left(x, \alpha_{0}, \ldots, \alpha_{n-1}\right) \Longleftrightarrow\left(\exists \alpha_{n}\right) P_{n+1}\left(x, \alpha_{0}, \ldots, \alpha_{n-1}, \alpha_{n}\right)$ (if $n$ is even).

(3) $P_{n}\left(x, \alpha_{0}, \ldots, \alpha_{n-1}\right) \Longleftrightarrow\left(\forall \alpha_{n}\right) P_{n+1}\left(x, \alpha_{0}, \ldots, \alpha_{n-1}, \alpha_{n}\right)$ (if $n$ is odd).

The basic idea of the proof is to define simultaneously a sequence of norms $\bar{\varphi}^{n}=$ $\left\langle\varphi_{i}^{n}: i \in \omega\right\rangle$ on all $P_{n}$ and then prove that each $\bar{\varphi}^{n}$ is a scale on $P_{n}$. We will naturally use the equivalences (2) and (3) and the constructions in the proof of the second periodicity theorem, 6C.1 and 6C.3 of [6]. The definition of $\varphi_{i}^{n}$ is by induction on $i$.

Basis. For each $n$, put

$$
\left.\varphi_{0}^{n}\left(x, \alpha_{0}, \ldots, \alpha_{n-1}\right)=0 \quad \text { (if } P_{n}\left(x, \alpha_{0}, \ldots, \alpha_{n-1}\right)\right) .
$$

This norm is trivial as far as its values are concerned, but it has a coinductive domain and it is easily seen to be a $\Sigma_{0}^{*}$-norm, and in general no better.

Induction step for even $n$. Let $\widetilde{\alpha}=\alpha_{0}, \ldots, \alpha_{n-1}$ to simplify notation, and using (2), put

$$
\begin{gathered}
\varphi_{i+1}^{n}(x, \widetilde{\alpha})=\operatorname{infimum}\left\{\left\langle\varphi_{0}^{n+1}\left(x, \widetilde{\alpha}, \alpha_{n}\right), \alpha_{n}(0), \varphi_{i}^{n+1}\left(x, \widetilde{\alpha}, \alpha_{n}\right), \alpha_{n}(1), \ldots,\right.\right. \\
\left.\varphi_{i}^{n+1}\left(x, \widetilde{\alpha}, \alpha_{n}(i)\right\rangle: P_{n+1}\left(x, \widetilde{\alpha}, \alpha_{n}\right)\right\}
\end{gathered}
$$

where of course $\langle, \ldots$,$\rangle is an order-preserving map of tuples of ordinals into$ the ordinals.

Induction step for odd $n$. With the same notation convention and using (3), we now put

$$
\begin{gathered}
\varphi_{i+1}^{n}(x, \tilde{\alpha})=\text { "supremum" }\left\{\left\langle\varphi_{0}^{n+1}\left(x, \widetilde{\alpha}, \alpha_{n}\right), \alpha_{n}(0), \varphi_{1}^{n+1}\left(x, \widetilde{\alpha}, \alpha_{n}\right), \alpha_{n}(1),\right.\right. \\
\left.\left.\ldots, \varphi_{i}^{n+1}\left(x, \widetilde{\alpha}, \alpha_{n}\right), \alpha_{n}(i)\right\rangle: \alpha \in N\right\},
\end{gathered}
$$

where of course we do not take the real supremum of the set of ordinals in braces, but the "fake supremum" determined by a game as in the proof of the second periodicity theorem $6 \mathrm{C} .3$ of [6]. (Without repeating that construction, it will suffice to recall that a game $G_{i+1}(x, \tilde{\alpha} ; y, \tilde{\beta})$ is defined for each $x, \tilde{\alpha}$ and $y, \tilde{\beta}$ in $P_{n}$ which involves the $(i+1)$ st sequence of numbers $u(i+1)$, the relation

$$
(x, \widetilde{\alpha}) \lesssim(y, \widetilde{\beta}) \Longleftrightarrow \text { II wins } G_{i+1}(x, \widetilde{\alpha} ; y, \widetilde{\beta})
$$


is shown to be a prewellordering on $P_{n}$, and $\varphi_{i+1}^{n}$ is simply the rank function of that prewellordering.)

We now have a sequence of norms $\bar{\varphi}^{n}$ on each $P_{n}$ and it is trivial to check that each $\varphi_{i}^{n}$ is a $\Sigma_{i}^{*}$-norm, uniformly; that $\bar{\varphi}^{0}$ is actually a scale on $P_{0}$ (as is each $\bar{\varphi}^{n}$ on $P_{n}$ ) follows by a careful analysis of the arguments in 6C.1 and 6C.3 of [6] which we will not put down here.

Martin has proved under the same determinacy hypothesis that this theorem is best possible in the following sense: a universal coinductive set does not admit a $\Sigma_{n}^{*}$-scale for any $n$.

2. The extent of scales in $L(R)$. Consequences of determinacy hypotheses are often formulated as results about the inner model $L(R)(=L(N))$, the smallest model of Zermelo-Fraenkel set theory which contains the reals $R$ and all the ordinals. There are many good reasons for this, including the obvious fact that if there is any inner model of AD (full determinacy) which contains $R$, then $L(R)$ is the smallest such model. It is generally believed that the hypothesis $L(R) \mid=$ $\mathrm{AD}$ should settle most set-theoretic problems about $L(R)$, just as ZF by itself settles most questions about $L$.

A pointset $P \subseteq X$ is $\Sigma_{1}^{2}$ in $L(R)$ if

where the relation $Q$ is definable in the language of set theory with all quantifiers restricted to $N$, so that it is absolute over $L(R)$.

Theorem B (Martin, Steel). A pointset $P$ is $\Sigma_{1}^{2}$ in $L(R)$ if and only if it is $\mathrm{N}^{2} \Pi_{1}^{1}$, i.e. if and only if it satisfies an equivalence of the form

$$
P(x) \Leftrightarrow\left\{\left(\exists \alpha_{0}\right)\left(\forall \alpha_{1}\right)\left(\exists \alpha_{2}\right) \ldots\right\} R\left(x,\left\langle\alpha_{0}, \alpha_{1}, \alpha_{2}, \ldots\right\rangle\right),
$$

where $R$ is a $\Pi_{1}^{1}$ pointset.

Outline of Proof. For the nontrivial direction, suppose $P$ is defined by (4) above and consider (for each $x$ ) the game $G(x)$ on $N$, where I plays $\alpha_{0}, \alpha_{1}, \ldots$, II plays $\beta_{0}, \beta_{1}, \ldots$ and in addition II assigns a truth value to all the sentences of set theory with $\alpha_{0}, \alpha_{1}, \ldots, \beta_{0}, \beta_{1}, \ldots$ as parameters; II wins if he defines in this way the truth set of a (countable) model $M$ whose reals are precisely $\alpha_{0}$, $\alpha_{1}, \ldots, \beta_{0}, \beta_{1}, \ldots$, which is well founded and which further satisfies the formal assertion

$$
\text { " } V=L(R) " \&(\exists A) Q(x, A) \&(\forall \xi)\left[L_{\xi}(R) \not(\exists A) Q(x, A)\right] .
$$

If $P(x)$ holds and $\kappa$ is the least ordinal such that $L_{\kappa}(R) \vDash(\exists A) Q(x, A)$, then II can win $G(x)$ by using Skolem functions for $L_{\kappa}(R)$; conversely, if II wins $G(x)$, then his strategy can be used to construct Skolem functions for some $L_{\kappa}(R)$ 
which satisfies $(\exists A) Q(x, A)$, so that we have

$$
P(x) \Longleftrightarrow \text { II wins } G(x) \text {. }
$$

The required representation for $P$ follows from this since $G(x)$ is clearly a $\Pi_{1}^{1}$ game.

Theorem C (MARtin, Steel). Assume $V=L(R) \&$ AD; then every $\Sigma_{1}^{2}$ pointset $P$ admits a $\Sigma_{1}^{2}$-scale and every pointset which admits a scale is $\Sigma_{1}^{2}$.

Outline of Proof. The second assertion was observed by Kechris and Solovay some time ago and follows (by a Wadge-type argument) from the fact that in $L(R)$ some $\Pi_{1}^{2}$ set cannot be uniformized (granting AD).

For the first assertion, by (5) above and the usual Shoenfield analysis of $\Pi_{1}^{1}$ sets, we get a representation of the form

$$
\begin{gathered}
\left.P(x) \Longleftrightarrow\left\{\exists \alpha_{0}\right)\left(\forall \alpha_{1}\right)\left(\exists \alpha_{2}\right) \ldots\right\}\left\{\left(\exists \xi_{0}\right)\left(\exists \xi_{1}\right)\left(\exists \xi_{2}\right) \ldots\right\} \\
(\forall t) S\left(x(t),\left\langle\alpha_{0}(t), \ldots, \bar{\alpha}_{t-1}(t)\right\rangle,\left\langle\xi_{0}, \ldots, \xi_{t-1}\right\rangle\right)
\end{gathered}
$$

for $\boldsymbol{P}$, and then by a simple open-game argument, we have

$$
\begin{aligned}
& P(x) \Longleftrightarrow\left\{\left(\exists \alpha_{0}\right)\left(\exists \xi_{0}\right)\left(\forall \alpha_{1}\right)\left(\exists \alpha_{2}\right)\left(\exists \xi_{1}\right)\left(\forall \alpha_{3}\right) \ldots\right\} \\
& (\forall t) S\left(x(t),\left\langle\alpha_{0}(t), \ldots, \bar{\alpha}_{t-1}(t)\right\rangle,\left\langle\xi_{0}, \ldots, \xi_{t-1}\right\rangle\right) ;
\end{aligned}
$$

now this is very similar to the representation $\left(1^{*}\right)$ for coinductive sets and the method of Theorem A extends easily to construct a scale on $P$. It is not too hard to verify further that this is a $\Sigma_{1}^{2}$-scale.

It is easy to check that in $L(R)$, every nonempty $\Sigma_{1}^{2}$ collection of pointsets must have a $\Delta_{1}^{2}$ member; thus by Theorem $\mathrm{C}$, granting $L(R) \vDash \mathrm{AD}$, if we can prove that every pointset $P$ which admits a scale satisfies a $\Pi_{1}^{2}$ assertion $\varphi(P)$, then $L(R) \vDash(\forall P) \varphi(P)$. Thus Theorem $\mathrm{C}$ lifts to all sets in $L(R)$ many of the structure and regularity results which are known for scaled sets - for example the Ramsey property.

3. Remarks and further results. Some of the arguments above (about games on $N$ ) appear to depend on the axiom of choice, but they can be easily reformulated in $\mathrm{ZF}+$ Dependent Choices by interpreting game-assertions in terms of multiple-valued strategies.

Steel has refined and extended considerably the proofs of Theorems A-C to obtain an almost complete analysis of the minimum complexity of a scale on a given pointset $P$ in $L(R)$, in terms of the fine structure of the constructible hierarchy $\left\langle L_{\xi}(R): \xi \in\right.$ Ordinals $\rangle$. In another direction, Martin has shown that with natural determinacy hypotheses for games on $N$ (stronger than $\mathrm{AD}$ ), the realgame quantifier $\mathrm{S}^{2}$ preserves scales and in fact so do the quantifiers which are 
associated in the same way with games on $N$ of any countable ordinal length. Martin's proof uses ideas from the proof of Theorem A and the third periodicity theorem $6 \mathrm{E} .1$ of $[6]$.

\section{BIBLIOGRAPHY}

1. A. S. Kechris and Y. N. Moschovakis, (eds.), Notes on the theory of scales, Cabal Seminar 76-77, Lecture Notes in Math., vol. 689, Springer-Verlag, Berlin and New York, 1978.

2. D. A. Martin and R. M. Solovay, A basis theorem for $\Sigma_{3}^{1}$ sets of reals, Ann. of Math. (2) 89 (1969), 138-160.

3. Y. N. Moschovakis, Uniformization in a playful universe, Bull. Amer. Math. Soc. 77 (1971), $731-736$. 1974.

4. - Elementary induction on abstract structures, North-Holland, Amsterdam,

5. Inductive scales on inductive sets, Cabal Seminar 76-77, Lecture Notes in Math. vol. 687, Springer-Verlag, Berlin and New York, 1978, pp. 185-192.

6. - Descriptive set theory, North-Holland, Amsterdam, 1980.

DEPARTMENT OF MATHEMATICS, UNIVERSITY OF CALIFORNIA, LOS ANGELES, CALIFORNIA 90024 